\title{
Fast magnetohydrodynamic oscillations in a multifibril Cartesian prominence model
}

\author{
A. J. Díaz ${ }^{\star}$, R. Oliver, and J. L. Ballester
}

\begin{abstract}
Departament de Física, Universitat de les Illes Balears, 07122 Palma de Mallorca, Spain e-mail: antonio@mcs.st-and.ac.uk; [ramon.oliver;dfsjlbQ]@uib.es
\end{abstract}

Received 25 January 2005 / Accepted 5 May 2005

\begin{abstract}
Observations of quiescent filaments show very fine structures which suggests that they can be composed of smallscale threads or fibrils. Two-dimensional, high-resolution observations point out that individual fibrils or groups of fibrils may oscillate independently with their own periods. In this paper, we study the fast magnetohydrodynamic modes of oscillation of multifibril Cartesian systems to represent the oscillations of the fibril structure of a real prominence. In the case of a system made of equal fibrils, our results show that the only non-leaky mode is the symmetric one, which means that all the fibrils oscillate in spatial phase with the same frequency. On the other hand, in a system made of non-equal fibrils, i.e. with different Alfvén speeds, the results show that the amplitudes of oscillation are higher in the denser fibrils, that the frequency of oscillation of the only non-leaky mode is slightly smaller than that of the dominant fibril considered alone, and that all the fibrils also oscillate in phase.
\end{abstract}

Key words. Sun: oscillations - Sun: magnetic fields - Sun: corona - Sun: prominences - Sun: filaments

\section{Introduction}

High-resolution observations of quiescent filaments show very fine structures within its body, suggesting that they are composed of small-scale threads. The existence of this internal structure in prominences was suggested by Menzel \& Evans (1953), was clarified with the improvement of observational capabilities (Engvold 1976; Engvold et al. 1987) and evidence for the existence of horizontal fine structures within prominences was later found (Schmieder \& Mein 1989; Schmieder et al. 1991; Lin et al. 2005). For instance, Engvold et al. (1987) observed a quiescent filament and, from the study of the prominence-corona interface, deduced that the fine structure of the cool filament core may consist of thin magnetic flux ropes oriented at an angle of $20^{\circ}$ to the filament long axis. Taking into account the observational evidence, magnetostatic equilibrium models for prominence fibrils have been constructed by Ballester \& Priest (1989), Degenhardt \& Deinzer (1993) and Schmitt \& Degenhardt (1996). These models represent a prominence fibril by means of a hot-cool loop modelled using the thin flux tube approximation.

On the other hand, the existence of small amplitude, periodic velocity oscillations in quiescent solar prominences is a well-known phenomenon (see Engvold 2001; Oliver \& Ballester 2002, for thorough reviews), and two-dimensional, high-resolution observations (Yi et al. 1991; Yi \& Engvold 1991; Engvold 2001; Lin 2004) have revealed that individual fibrils or groups of fibrils may oscillate independently with

* Present address: Mathematical Institute, University of St. Andrews, St. Andrews, KY16 9SS, Scotland, UK. their own periods, which lie between 3 and 20 min. Hence, one of the basic questions in prominence seismology is whether periodic changes in prominences are always associated with their fibril structure or not.

The first theoretical investigation of periodic prominence perturbations taking into account the prominence fine structure was performed using Cartesian geometry by Joarder et al. (1997). Díaz et al. (2001, hereafter Paper I) have done a more in-depth analytical and numerical study of this type of configuration and the most important conclusions extracted from this study are that prominence fibrils can only support a few modes of oscillation (those with the smallest frequency, since high harmonics cannot be trapped inside the thin loop) and that the spatial structure of the fundamental even and odd kink modes is such that the velocity amplitude outside the fibril is still significant over long distances, i.e. the energy confinement in the fibril is rather poor. An interesting consequence is that fibrils may actually oscillate in groups rather than individually. However, the study in Paper I only considered a single, isolated fibril, thus neglecting the interaction among fibrils that should be expected in a real prominence.

Motivated by the observations suggesting that groups of fibrils may oscillate together, our main goal in this paper is to study the interactions between oscillating fibrils. Following the same procedure as in Paper I, we use Cartesian geometry, although we are aware that a more accurate description of prominence fibrils requires the use of cylindrical geometry.

The paper is organised as follows: in Sect. 2 the equilibrium model, the basic assumptions, the fast wave equations as well 


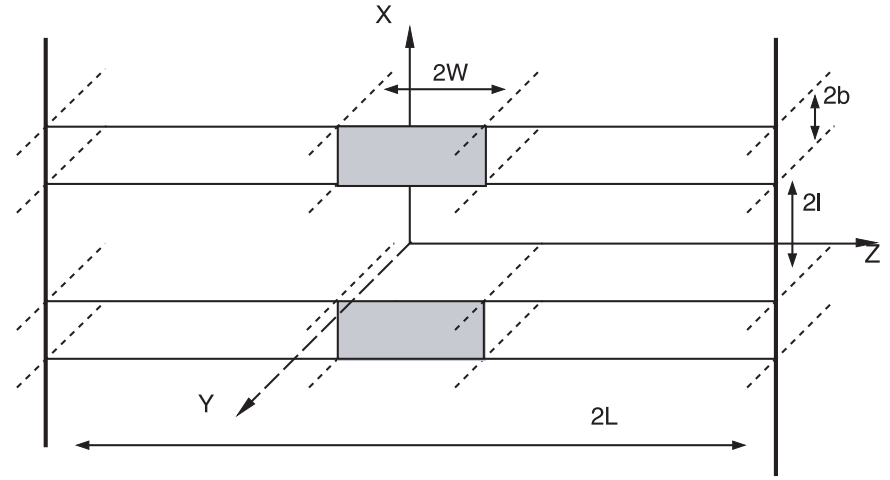

Fig. 1. The equilibrium configuration used in this study of a system of two fibrils. The grey zone represents the cold part of the slabs, i.e. the prominence. The density in the prominence region is $\rho_{\mathrm{p}}$, in the evacuated (coronal) part of the slab, $\rho_{\mathrm{e}}$, and in the coronal environment, $\rho_{\mathrm{c}}$. The magnetic field is uniform and parallel to the $z$-axis, and the whole configuration is invariant in the $y$-direction.

as their analytical solution are described. In Sect. 3 we present and discuss the results obtained when a couple of fibrils are considered, while in Sect. 4 the numerical solution for the case of several fibrils is considered. In Sect. 5 our conclusions are presented.

\section{Model, basic equations and analytical solution}

The equilibrium model consists of a collection of fibrils separated a distance $2 l$ (which is the relevant parameter and can differ between pairs when more that two fibrils are considered). Each fibril is then modelled as in Paper I: a straight slab of total length $2 L$ made of a cold and dense part (the prominence fibril itself) with length $2 W$ and density $\rho_{\mathrm{p}}$, and a hotter, coronal gas with density $\rho_{\mathrm{e}}$ occupying the rest of the slab. The thickness of the structure is $2 b$ and it is embedded in the coronal environment, with density $\rho_{\mathrm{c}}$. The slab is anchored in the photosphere, so its footpoints are subject to line-tying conditions. Finally, the plasma is permeated by a uniform magnetic field directed along the prominence fibril. Because gravity is neglected, all other physical variables $(\rho, T$ and $p)$ are also uniform in each of the three regions. We also assume invariance in the $y$-direction. A sketch of the model for two fibrils is plotted in Fig. 1.

\subsection{Perturbation wave equations}

To derive a wave equation for this model we follow the procedure explained in Díaz et al. (2002). We consider a uniform, static plasma with unperturbed density $\rho_{0}$ and equilibrium magnetic field $\boldsymbol{B}_{0}=B_{0} \hat{\boldsymbol{e}}_{z}$. Next, linear, adiabatic perturbations about this equilibrium are introduced and the magnetic field and pressure perturbations, $\boldsymbol{B}$ and $p$, are eliminated in favour of the velocity and the total pressure perturbations, $\boldsymbol{v}$ and $p_{\mathrm{T}}$, so the following equations are obtained (Roberts 1991),

$\frac{\partial p_{\mathrm{T}}}{\partial t}=\rho_{0} c_{\mathrm{A}}^{2} \frac{\partial v_{z}}{\partial z}-\rho_{0} c_{\mathrm{f}}^{2} \nabla \cdot \boldsymbol{v}$,

$\rho_{0}\left(\frac{\partial^{2}}{\partial t^{2}}-c_{\mathrm{A}}^{2} \frac{\partial^{2}}{\partial z^{2}}\right) \boldsymbol{v}_{\perp}+\nabla_{\perp} \frac{\partial p_{\mathrm{T}}}{\partial t}=0$ $\rho_{0}\left(\frac{\partial^{2}}{\partial t^{2}}-c_{\mathrm{T}}^{2} \frac{\partial^{2}}{\partial z^{2}}\right) v_{z}+\frac{c_{\mathrm{s}}^{2}}{c_{\mathrm{f}}^{2}} \frac{\partial}{\partial z}\left(\frac{\partial p_{\mathrm{T}}}{\partial t}\right)=0$,

where the symbol $\perp$ stands for the components of the perturbed velocity and the gradient perpendicular to $\boldsymbol{B}_{0}, c_{\mathrm{s}}$ is the sound speed, $c_{\mathrm{A}}$ the Alfvén speed and the other characteristic speeds are defined as $c_{\mathrm{f}}^{2}=c_{\mathrm{s}}^{2}+c_{\mathrm{A}}^{2}$ and $c_{\mathrm{T}}^{-2}=c_{\mathrm{S}}^{-2}+c_{\mathrm{A}}^{-2}$.

Next, we consider the limit $\beta \rightarrow 0$, which implies that the slow mode is absent: the component of the perturbed velocity along the magnetic field, $v_{z}$, is identically zero from Eq. (3). Using $p_{\mathrm{T}}$ as main dependent variable we derive the following equation,

$\left(\frac{\partial^{2}}{\partial t^{2}}-c_{\mathrm{A}}^{2} \nabla^{2}\right) p_{\mathrm{T}}=0$

which is solved together with Eq. (2), necessary for some of the boundary conditions. We are only interested in stationary perturbations, so in what follows a temporal dependence of the form $\mathrm{e}^{-\mathrm{i} \omega t}$ is considered.

\subsection{Analytical solution for two identical fibrils}

The aim is to solve Eqs. (2) and (4) with the appropriate boundary conditions for the two-fibril system sketched in Fig. 1. The standard method for solving them in a closed region is separation of variables, $p_{\mathrm{T}}(x, z)=u(x) h(z)$, which gives us two ordinary differential equations (since longitudinal propagation is not considered),

$$
\begin{aligned}
& \frac{\mathrm{d}^{2}}{\mathrm{~d} x^{2}} u(x)=\lambda^{2} u(x), \\
& \left(\frac{\mathrm{d}^{2}}{\mathrm{~d} z^{2}}+\frac{\omega^{2}}{c_{\mathrm{A}}^{2}}\right) h(z)=-\lambda^{2} h(z) .
\end{aligned}
$$

These equations must be solved in three regions (numbered 1, 2 and 3, respectively): the coronal environment $(|x|>2 b+l)$, the fibrils $(l>|x|>2 b+l)$ and the region between them $(|x|<l)$, whose physical properties are supposed to be identical to the coronal ones. Therefore, our solution can be written as

$$
p_{\mathrm{T}}(x, z)= \begin{cases}\sum_{n=1}^{\infty} u_{n}^{(1)}(x) h_{n}^{(1)}(z), & |x| \geq 2 b+l, \\ \sum_{n=1}^{\infty} u_{n}^{(2)}(x) h_{n}^{(2)}(z), & l \leq|x|<2 b+l, \\ \sum_{n=1}^{\infty} u_{n}^{(3)}(x) h_{n}^{(3)}(z), & 0 \leq x \mid<l,\end{cases}
$$

in which the need to use all the basis functions to fulfil the boundary conditions on the fibril surfaces has been taken into account (see Paper I for a discussion of this issue). Now, the $z$-dependent functions of Eq. (6) are calculated, taking into account the line-tying at $z= \pm L$ and the jump conditions at $z= \pm W$ (for the slab region). Notice also that the coronal medium is the same in regions 1 and 3 , so $h_{n}^{(1)}(z)=h_{n}^{(3)}(z)$, and using the same properties of Paper I the inner basis functions can be expressed in terms of the outer ones in the form $h_{n}^{(2)}(z)=\sum_{m=1}^{\infty} H_{n m} h_{n}^{(1)}(z)$, in which the coefficients $H_{n m}$ are the ones calculated in Paper I. 
Since a system made of equal fibrils is under consideration, the equations can be solved in the region $x \geq 0$ and $0 \leq z \leq L$, so there are even and odd modes in the $z$-direction and even and odd modes in the $x$-direction. The even modes in the $x$ direction are those in which both fibrils oscillate in phase and hereafter we call them symmetric modes, while the odd ones are antisymmetric modes. Taking into account these symmetries, the solution of the $x$-dependent part for a symmetric mode is of the form

$$
\begin{array}{rlrl}
u_{n}^{(1)}(x) & =A_{n} \mathrm{e}^{-\lambda_{n}^{(1)}(x-2 b-l),} & & x \geq 2 b+l, \\
u_{n}^{(2)}(x) & =B_{n}^{\sin } \sin \lambda_{n}^{(2)}(x-l) & & \\
\quad+B_{n}^{\cos } \cos \lambda_{n}^{(2)}(x-l), & & l \leq x<2 b+l, \\
u_{n}^{(3)}(x) & =C_{n} \cosh \lambda_{n}^{(3)} x, & & 0 \leq x<l .
\end{array}
$$

The $z$-dependent part depends on the Alfvén speed profile along the fibril. Following the model in Edwin \& Roberts (1982), we could take a cold slab where these functions are just $h_{n}^{(1)}(z)=$ $h_{n}^{(2)}(z)=h_{n}^{(3)}(z)=L^{-1 / 2} \cos \pi(2 n-1) z /(2 L)$, and $H_{n m}=\delta_{n m}$, so the modes of two cold Cartesian slabs could be studied, but here we will work directly with our model, and these functions are the same as in Paper I for the corona (numbered 1 and 3 here) and the fibril (numbered 2).

The next step is to apply the boundary conditions at $x=l$ and $x=2 b+l$, that is, continuity of the total pressure and of the $x$-components of the perturbed velocity and magnetic field, namely

$\boldsymbol{n} \cdot[\boldsymbol{v}]=0, \boldsymbol{n} \cdot[\boldsymbol{B}]=0, \quad\left[p_{\mathrm{T}}\right]=0$.

It is useful to deduce an expression of the component of the perturbed velocity in the $x$-direction from Eq. (2):

$v_{x}(x, y, z)=\frac{\mathrm{i} \omega}{\rho_{0} c_{\mathrm{A}}^{2}} \sum_{n=1}^{\infty} \frac{1}{\lambda_{n}^{2}} \frac{\mathrm{d} u_{n}(x)}{\mathrm{d} x} h_{n}(z)$.

Operating with these expressions the coefficients $A_{n}$ and $C_{n}$ in Eq. (8) can be eliminated, and we obtain for the symmetric modes

$$
\begin{aligned}
& \sum_{l=0}^{\infty} H_{l n} {\left[B_{l}^{\cos } \lambda_{n}^{(3)} \sinh \lambda_{n}^{(3)} l-B_{l}^{\sin } \lambda_{n}^{(2)} \cosh \lambda_{n}^{(3)} l\right]=0 } \\
& \sum_{l=0}^{\infty} H_{l n}\left[\lambda_{n}^{(1)}\left(B_{l}^{\sin } \sin \lambda_{n}^{(2)} 2 b+B_{l}^{\cos } \cos \lambda_{n}^{(2)} 2 b\right)\right. \\
&\left.\quad+\lambda_{n}^{(2)}\left(B_{l}^{\sin } \cos \lambda_{n}^{(2)} 2 b-B_{l}^{\cos } \sin \lambda_{n}^{(2)} 2 b\right)\right]=0
\end{aligned}
$$

and similar expressions for the antisymmetric ones.

The above equations constitute two infinite systems of homogeneous algebraic equations for the coefficients $B_{l}^{\text {sin }}$ and $B_{l}^{\text {cos }}$, with coefficients that depend on $\omega$. The condition to have a non-trivial solution is that the determinant of this system vanishes, providing us with the dispersion relation from which the eigenvalues of the problem can be obtained. Obviously, an infinite determinant is extremely difficult to treat, so it is truncated by taking $B_{s}=0$ for $n>N$. Thus, we are left with a $2 N$-order determinant, obtained by using the first $N$ basis functions only (with $N$ large, at least 24). Once the eigenfrequencies have been obtained, all the other perturbed quantities in the problem can be determined.

This system only has a non-trivial solution if some of the $\lambda_{n}^{(2)}$ are allowed to become complex. In this case, the $x$ dependence inside the fibril is in the form of hyperbolic functions instead of trigonometric ones in Eq. (11).

The solution method developed in this subsection can also be applied with minor changes to other systems, such as two non-equal fibrils or more than two of them. However, the resulting system of equations is much more complicated and difficult to treat than Eqs. (11). For that reason, the finite-differences numerical code described in Arregui et al. (2001, 2003) has been used. It solves the resulting partial differential wave equations for the perturbed velocity, although the perturbed pressure can also be obtained as an output. The agreement with the analytical solution is extremely good in the problem of two fibrils and, moreover, the numerical program reproduces even the step discontinuities in the solution due to the MHD boundary conditions.

\section{Two-fibril system}

There is no reason to assume that all the fibrils composing a prominence are identical. In fact, observations of prominences suggest that they are highly non-homogeneous in density and temperature, while the possible inhomogeneity in magnetic field is much more difficult to confirm. However, first of all, we can study the interaction among identical fibrils conforming a homogeneous prominence to investigate the new features in the most simple example. Then, a similar study will be numerically performed for the case of non-identical fibrils i.e. for a non-homogeneous prominence.

\subsection{Identical fibrils: dependence of the modes on the fibril separation}

For this study, the key parameter is $l$, half the distance between the two fibrils. Notice that in the limit $l \rightarrow \infty$, two separate fibrils are recovered (and both the sausage and kink modes from Paper I are present), while in the limit $l \rightarrow 0$ the fibrils coalesce and form a single one with width $4 b$ and, as before, the sausage and kink modes corresponding to this situation are present.

First of all, in Fig. 2 the variation of the frequency for symmetric and antisymmetric modes is shown for two different sets of parameters. From Paper I it can be easily checked that the limits $l \rightarrow \infty$ and $l \rightarrow 0$ are correctly described, and when the fibrils are separated by more than twice their width ( $l>2 b$ approximately) there is no coupling between them, i.e. the symmetric and antisymmetric modes are equivalent in frequency and correspond to those of a single fibril oscillating alone. When the two fibrils are closer, the interaction is clearly shown and the two modes become separated in frequency. In the limit $l \rightarrow 0$ the frequency of the symmetric modes is smaller than that of the antisymmetric ones and, because of the interaction, the modes can cross the cut-off frequency. As an example, in Fig. 2a, the fourth symmetric mode, which is under the cutoff frequency for a fibril having $b / L=0.5$ (limit $l \rightarrow 0$ ) but 

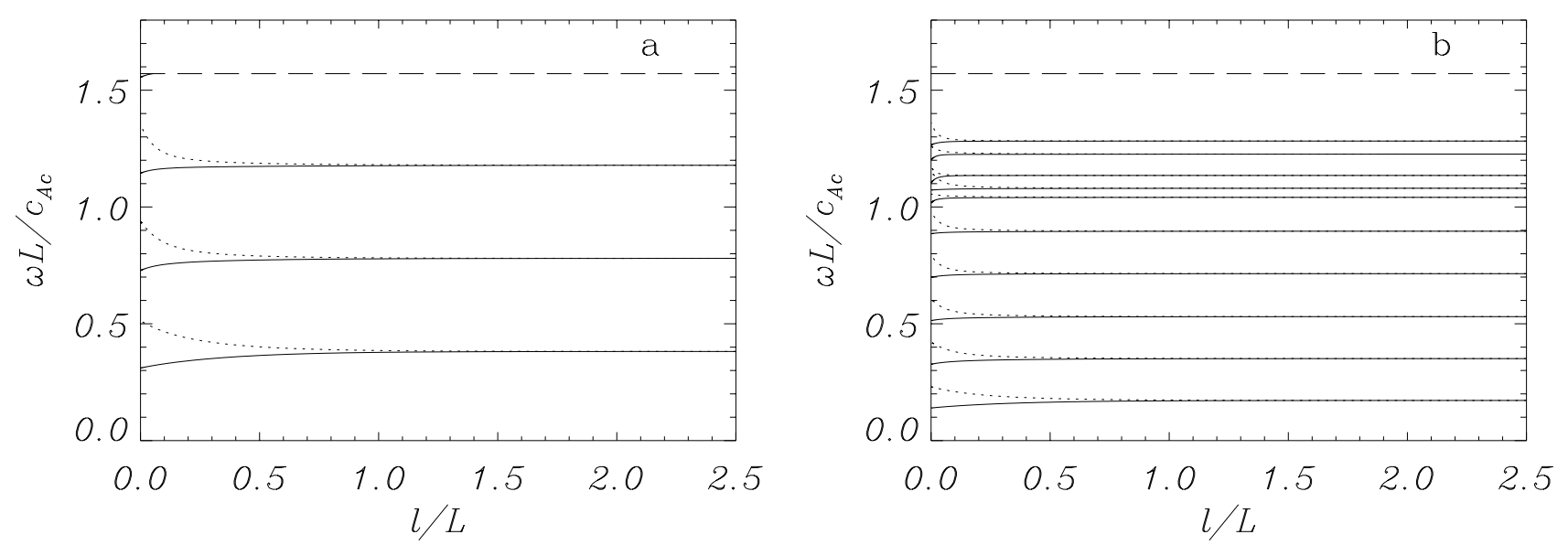

Fig. 2. Two identical fibrils: variation of the frequency with the half-separation for symmetric even modes (solid lines) and antisymmetric even modes (dotted lines) for two different sets of parameters: a) $W / L=0.1, \rho_{\mathrm{e}} / \rho_{\mathrm{c}}=0.6, \rho_{\mathrm{p}} / \rho_{\mathrm{c}}=200$ and $\left.b / L=0.5 ; \mathbf{b}\right) W / L=0.1, \rho_{\mathrm{e}} / \rho_{\mathrm{c}}=1$, $\rho_{\mathrm{p}} / \rho_{\mathrm{c}}=1000$ and $b / L=0.5$ (only the 10 first eigenfunctions in each symmetry). Notice that in a) the fourth symmetric mode appears from the cut-off (dashed line) when the fibrils get closer.
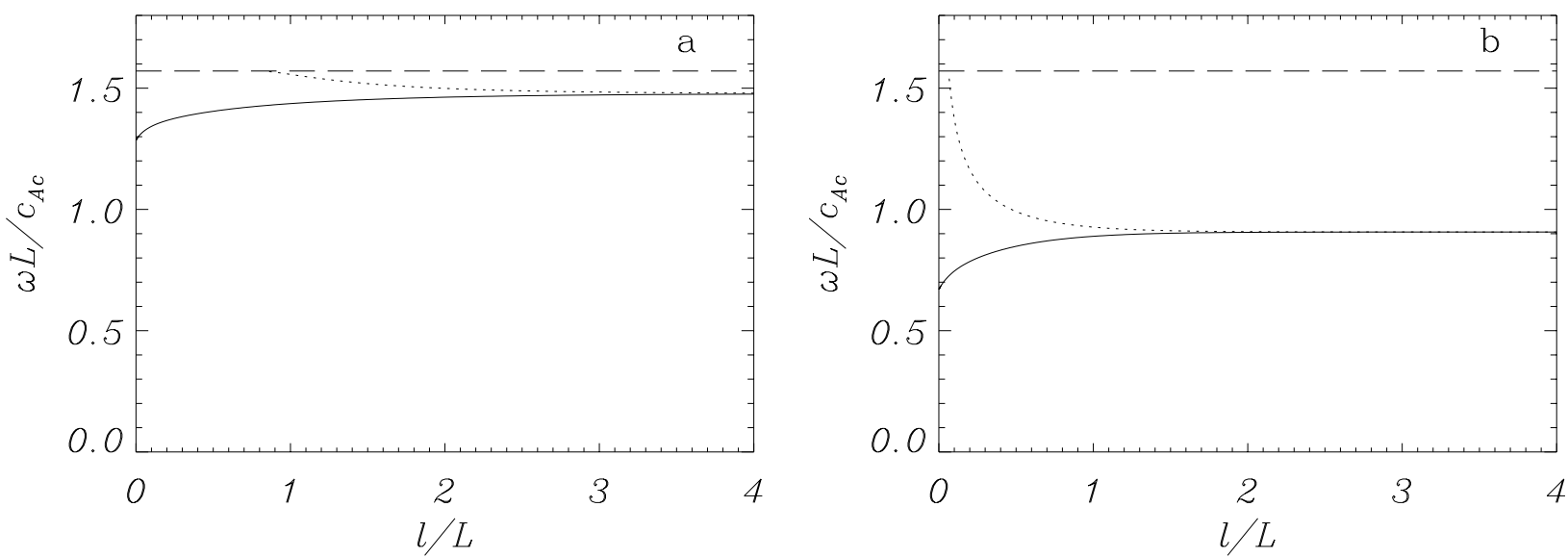

Fig. 3. Two identical fibrils: variation of frequency with the half-separation for symmetric even modes (solid lines) and antisymmetric even modes (dotted lines) for two sets of parameters: a) $W / L=0.1, \rho_{\mathrm{e}} / \rho_{\mathrm{c}}=0.6, \rho_{\mathrm{p}} / \rho_{\mathrm{c}}=200$ and $\left.b / L=0.005 ; \mathbf{b}\right) W / L=0.1, \rho_{\mathrm{e}} / \rho_{\mathrm{c}}=1$, $\rho_{\mathrm{p}} / \rho_{\mathrm{c}}=1000$ and $b / L=0.005$. Now there is only one surviving mode in each symmetry. Notice that the antisymmetric mode has a cut-off (dashed line) for low values of $b / L$.

is over the cut-off for a single fibril of width $b / L=0.25$ (limit $l \rightarrow \infty$ ), appears for low values of the parameter $l$ and becomes leaky when $l$ is increased.

On the other hand, note the complex interaction of modes shown in Fig. 2b (larger density contrast, $\rho_{\mathrm{p}} / \rho_{\mathrm{c}}$ ). A mode can cross other modes with different types of symmetry due to the coupling, but not modes having the same symmetry. The spacing between the modes is not regular, because there are space couplings for this set of parameters (see Paper I). It is also noticeable in both Figs. $2 \mathrm{a}$ and $2 \mathrm{~b}$ that the higher the order of the mode, the later the interaction between symmetric and antisymmetric modes starts. The explanation for this behaviour is that even in a single fibril, the modes with higher orders are more spatially confined, so the fibrils must be closer to feel their mutual interaction.

The mentioned above features only appear when the thickness of the fibrils is far from the realistic range $b / L \approx$ $0.001-0.01$. For this situation, the behaviour is illustrated in Fig. 3. There are few confined modes left and decreasing the parameter $b / L$ there would be only one left with its frequency tending to $\pi / 2$ (see Paper I). In this case, the influence of both fibrils on the frequency is noticeable even for high values of the separation $2 l / L$ compared to the fibril width $b / L$ (in our case, over 500 times), so in this model two prominence fibrils would always interact. Furthermore, the antisymmetric mode becomes leaky when the two fibrils become closer, as can be clearly seen in Fig. 3. Hence, the system is constrained to oscillate in phase if realistic values of the parameters are used.

\subsection{Identical fibrils: spatial profiles}

We now investigate the spatial properties of the modes. The solution for the $x$-component of the perturbed velocity is plotted in Fig. 4, where the fibrils are wide and the interaction is strong enough to differentiate very well symmetrical and antisymmetrical modes in the spatial structure (and even in frequency). It can also be checked that the dependence in the $z$-coordinate is nearly the same as for one fibril alone (described in Paper I), 


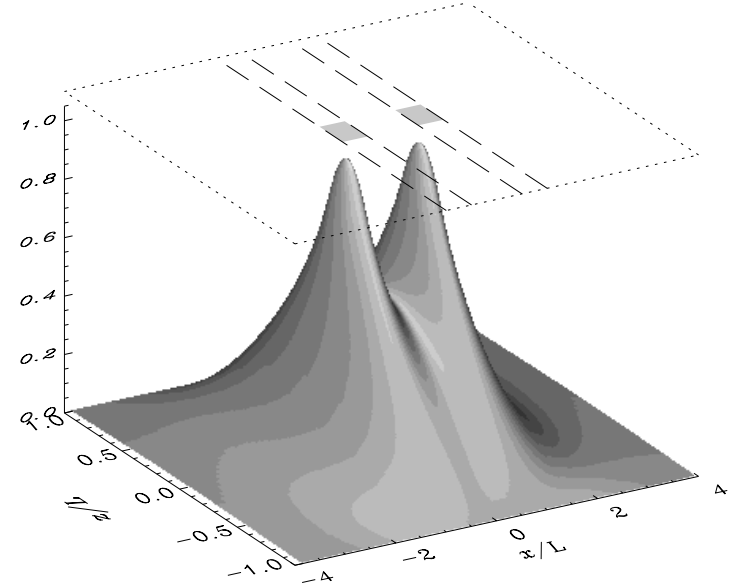

(a)

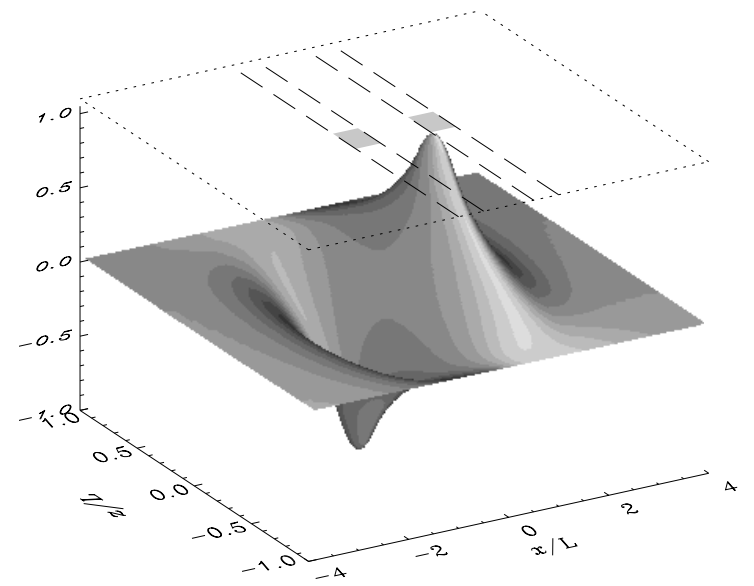

(b)

Fig. 4. Two identical fibrils: a) symmetric; and b) antisymmetric first mode for two wide fibrils with parameters $W / L=0.1, \rho_{\mathrm{e}} / \rho_{\mathrm{c}}=0.6$, $\rho_{\mathrm{p}} / \rho_{\mathrm{c}}=200, b / L=0.5$ (Fig. 2) and half separation $l / L=0.5$. In the plane $z=1.1$ a sketch of the equilibrium model has been included, with the fibril region shaded in grey. Notice that the whole range in the $z$ direction has been plotted, and also that the two fibrils are included in these surface plots.
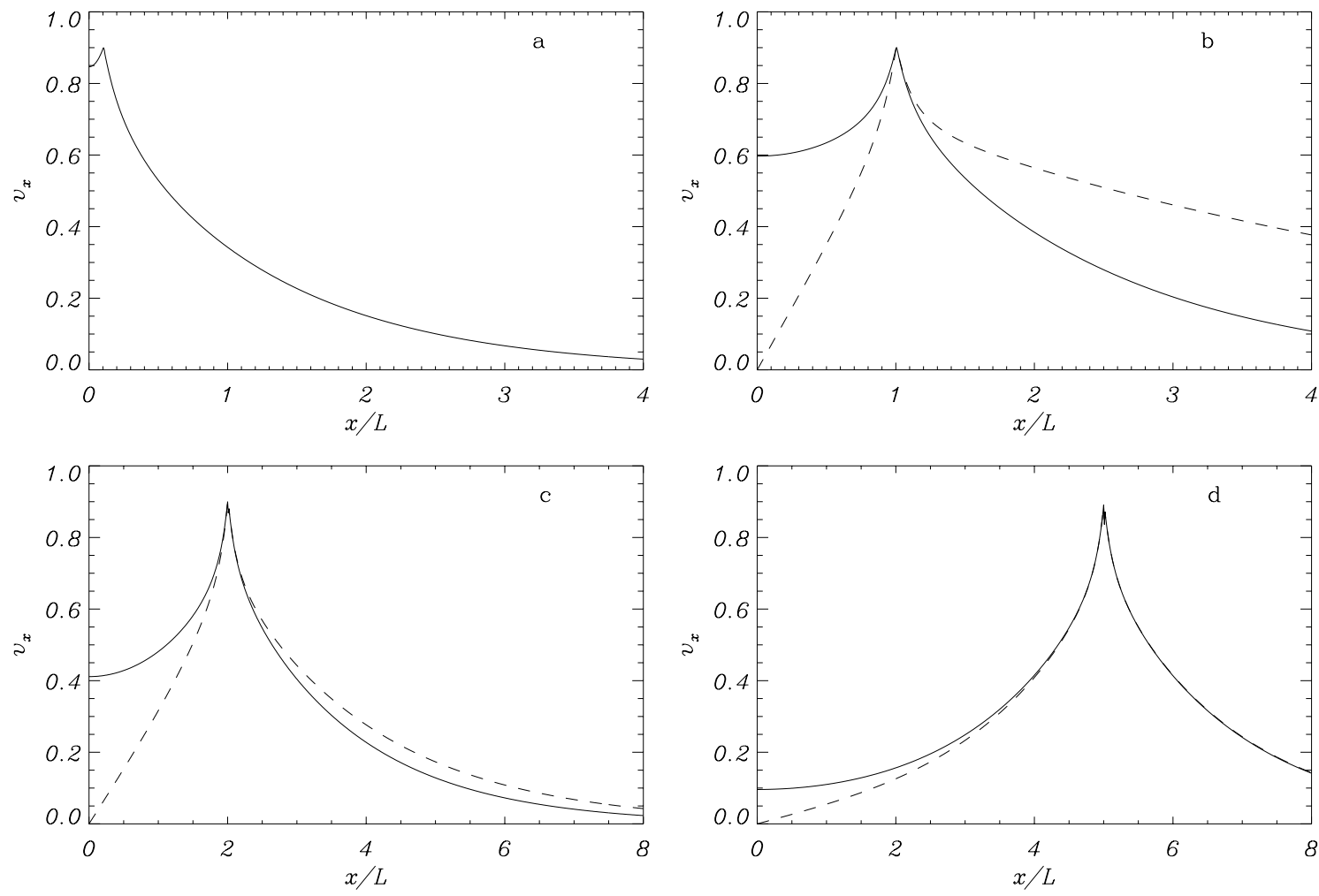

Fig. 5. Two identical fibrils: cuts of $v_{x}$ in the direction $z=0$ of the first symmetric (solid line) and antisymmetric (dashed line) mode for the parameters $W / L=0.1, \rho_{\mathrm{e}} / \rho_{\mathrm{c}}=0.6, \rho_{\mathrm{p}} / \rho_{\mathrm{c}}=200, b / L=0.01$ (Fig. 3) and a) $l / L=0.1$, b) $l / L=1$, c) $l / L=2$ and d) $l / L=5$. Notice that the $x$-scale is changed in the last two panels, while in the first one, the sausage mode has passed through a cut-off (i.e. has become leaky).

so we will concentrate on the features concerning the spatial profiles across the fibrils.

Let us move now to realistic parameters. Figure 5 displays the evolution of the spatial structure of the two surviving modes when the distance between the fibrils is increased (only the region $x \leq 0$ is represented, since it is easy to reconstruct the whole solution due to the symmetry of the modes). In Fig. 5a, only the symmetric mode is present, so both fibrils oscillate in spatial phase. In Fig. 5b, the antisymmetric mode is also present, but just under the cut-off frequency, and therefore it extends to distances far away from the system. In Fig. 5c, the two modes become closer in frequency and also in spatial shape, and finally in Fig. 5d, they are quite similar.

From Fig. 5d it is also clear how the single fibril modes are recovered. Adding these modes, the amplitude in the second fibril (that corresponds to $x / L=-5$, not shown in the graph) 


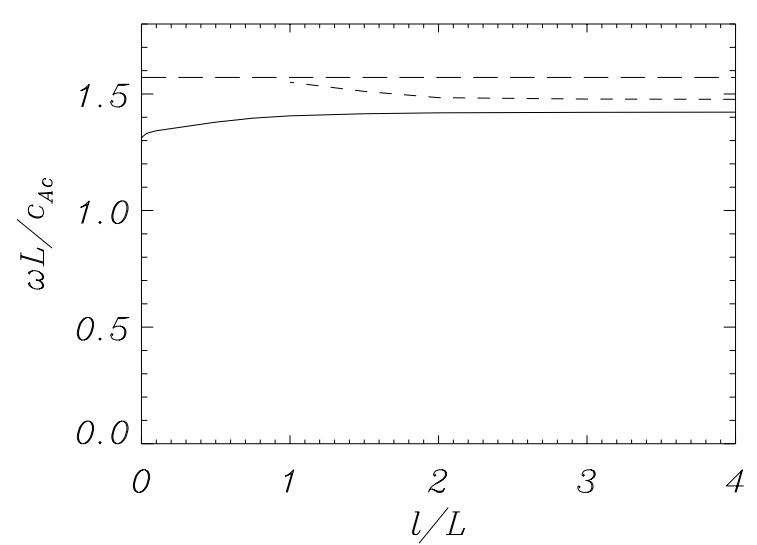

Fig. 6. Dispersion relation for two non-identical fibrils for the set of parameters $W / L=0.1, \rho_{\mathrm{e}} / \rho_{\mathrm{c}}=0.6, b / L=0.005$ and $\rho_{\mathrm{p}} / \rho_{\mathrm{c}}=200$ for one of them and $\rho_{\mathrm{p}} / \rho_{\mathrm{c}}=150$ for the other. The solid and dashed lines are the symmetric and antisymmetric modes respectively.

vanishes, and what is left is just the first one oscillating on its own (with a tiny contribution of the second one). If the second fibril was pushed further away, the single fibril results would be recovered exactly.

\subsection{Non-identical fibrils}

We now investigate the eigenmodes of non-identical fibrils; this is done numerically in order to avoid cumbersome calculations. The difference between the fibrils is their density, which modifies the Alfvén velocity within the fibril.

The first difference from the case of identical fibrils is that in the limit of high separation there are not two degenerate modes (symmetric and antisymmetric), but two fibrils oscillating alone and each fibril having its own eigenfrequency. As the fibrils come closer the modes interact, and the mode coming from the denser fibril becomes the one in which both fibrils vibrate in phase (similar to a symmetric mode), while the mode coming from the less dense fibril becomes the mode in which both fibrils vibrate in opposition of phase (similar to an antisymmetric mode). For a realistic value of the fibril thickness, the antisymmetric mode becomes leaky when the fibril separation is reduced, especially for the values that might be expected in solar prominences. This behaviour is illustrated in Fig. 6.

Regarding the spatial structure, it can be seen in Fig. 7 that for the symmetric mode (Fig. 7a) the perturbed velocity is higher in the denser fibril, while for the antisymmetric mode (Fig. 7b) it is higher in the less dense one. When the fibrils are separated by a distance of the order of their thickness the spatial structure is similar to that of a single fibril (Fig. 8a) oscillating with a frequency slightly smaller than that of the dense fibril. However, if we zoom on the fibril region (Fig. 8b) we see that there are small bumps in the regions of dense material.

Therefore, if the fibrils are not equal, for long separations between them there are important differences from the system of identical fibrils: the modes do not tend to a single frequency but to the values corresponding to the fibrils alone. Their spatial structure is also different, with velocity perturbations mainly in one of the fibrils (because the symmetry of the previous subsections is lost), but for realistic values of the thickness and separation, the interaction is very strong, so the fibrils end up oscillating in phase with velocity perturbations far away from them, with minor changes from the identical fibrils case.

\section{Numerical analysis of multifibril systems}

\subsection{Homogeneous prominence}

The analytical method described in Sect. 2.2 can be extended with no theoretical difficulty to the study of systems with more than two fibrils. However, the system of equations akin to Eq. (11) becomes too cumbersome and, therefore, we numerically solve the partial differential equation for the perturbed velocity component.

For simplicity, we first study systems of $n_{\mathrm{f}}$ identical fibrils having thickness $2 b$, with a separation $2 l$ between consecutive fibrils. With these conditions, and in the limit of no interaction ( $l / L$ large enough) there are as many modes with the same frequency as fibrils, and for $l \rightarrow 0$ there is a single oscillating fibril with thickness $n_{\mathrm{f}} 2 b$ (both limits can be calculated with the techniques of Paper I).

The resulting dispersion relations for four fibrils can be seen in Fig. 9. The main conclusion of this graph is that for realistic separations of fibrils (that is, $l \approx b$ ) there is only one non-leaky mode (the first symmetric one, in which all the fibrils oscillate in phase), while the modes with fibrils oscillating in opposition become leaky for large values of $l$. Moreover, the frequency of the confined mode is very close to that of a single fibril with width $n_{\mathrm{f}} 2 b$ (for the figure, the results in Paper I give $\omega(b / L=0.02)=1.019 c_{\mathrm{A}} / L$, which is the numerical value of Fig. 9 in the limit $l / L \rightarrow 0$ ).

Regarding the spatial structure, the perturbed velocity component around the fibril region is plotted in Fig. 10 for the system of four identical fibrils. This panel shows the velocity spatial structure in and near the fibrils and, since the interaction is very strong ( $l / L$ small), the global shape approaches that for a single fibril having width $n_{\mathrm{f}} 2 b$. Thus, the overall perturbation is similar to the one in Fig. 8a, with a peak in the prominence region and a slow decay away from it, but if we zoom on that region all the fibrils are marked as a small bumps, as can be seen in Fig. 10, but they oscillate with just slightly more amplitude than the surrounding coronal medium.

\subsubsection{Non-homogeneous prominence}

The above model can be applied to more complex configurations such as those expected to be found in real filaments. As an example, we study the oscillatory modes of the structure plotted in Fig. 11, in which all the fibrils are inside flux tubes such as those described in Fig. 1. The different fibril density ratios represent the density inhomogeneity of a real prominence, i.e. a different Alfvén velocity for each fibril, and the thickness and separation between fibrils have been chosen randomly within a realistic range.

We obtain the eigenmodes of this configuration. When the separation between fibrils is that shown in Fig. 11, there is a strong interaction between them since the perturbation can 

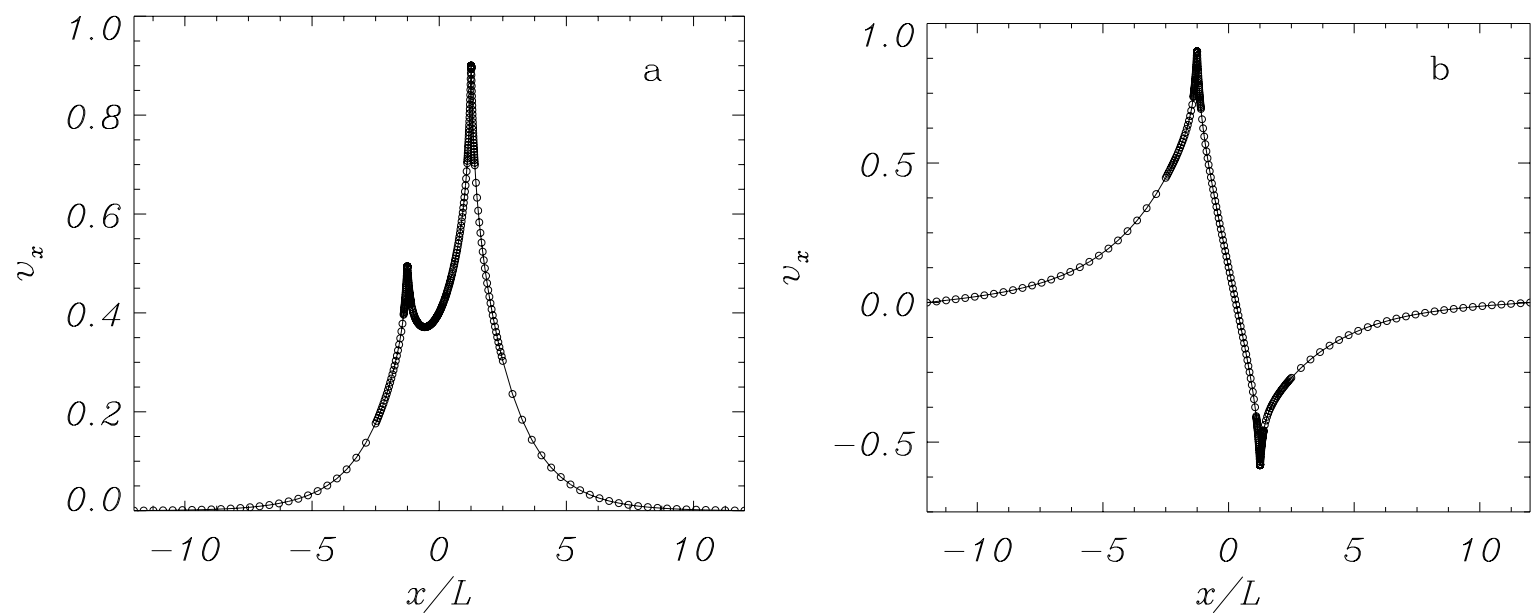

Fig. 7. Cuts in the direction $z=0$ for two non-identical fibrils for the set of parameters $W / L=0.1, \rho_{\mathrm{e}} / \rho_{\mathrm{c}}=0.6, b / L=0.005, l / L=1.25$ and density $\rho_{\mathrm{p}} / \rho_{\mathrm{c}}=200$ for the one situated at $x / L=1.25$ and $\rho_{\mathrm{p}} / \rho_{\mathrm{c}}=150$ for the one at $x / L=-1.25$. Panel a) is a symmetric mode with $\omega L / c_{\mathrm{A}}=1.412$; and panel $\mathbf{b}$ ) an antisymmetric mode with $\omega L / c_{\mathrm{A}}=1.525$, which is near the cut-off frequency.
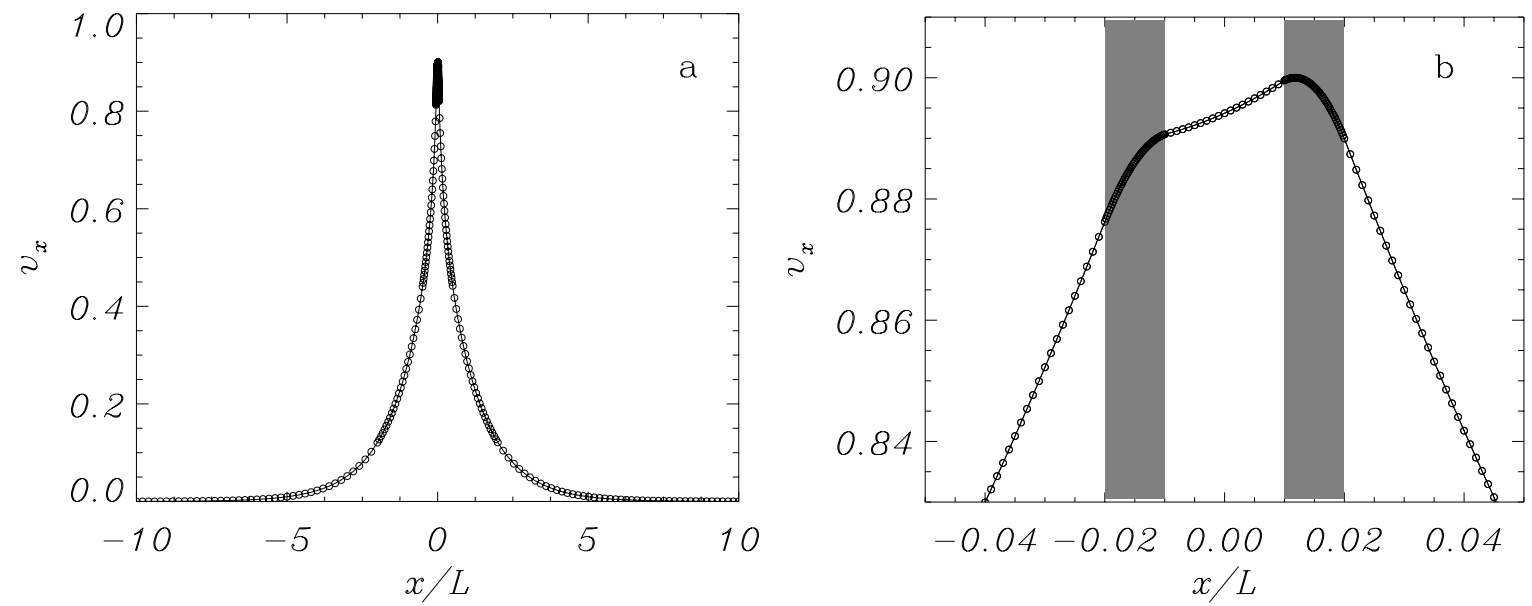

Fig. 8. a) Cut in the direction $z=0$ for the symmetric mode $\left(\omega L / c_{\mathrm{A}}=1.317\right)$ of two non-identical fibrils for the set of parameters $W / L=0.1$, $\rho_{\mathrm{e}} / \rho_{\mathrm{c}}=0.6, b / L=0.005, l / L=0.01, \rho_{\mathrm{p}} / \rho_{\mathrm{c}}=200$ for one situated at $x / L=0.01$ and $\rho_{\mathrm{p}} / \rho_{\mathrm{c}}=150$ for the one at $x / L=-0.01$. b) Detail of the zone around the fibrils (shaded regions).

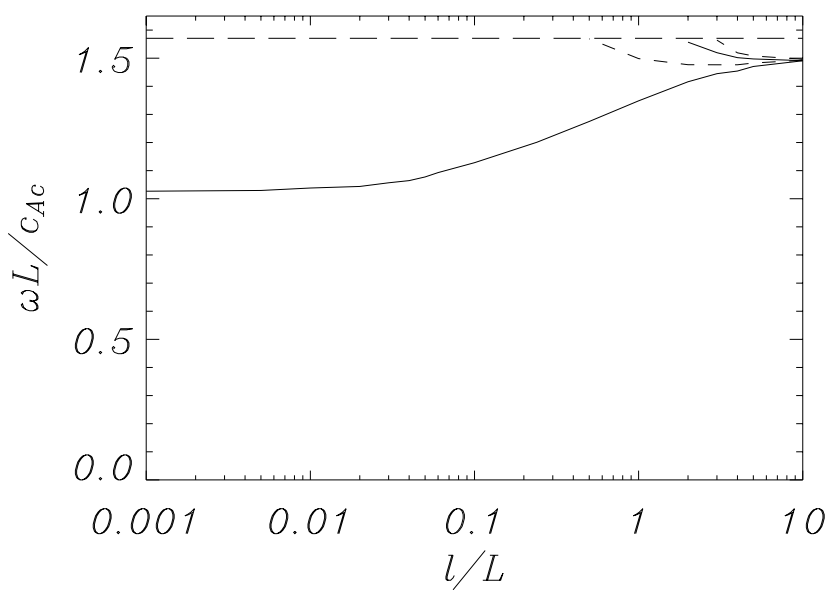

Fig. 9. Logarithmic plot of the dispersion relation for four identical fibrils for the set of parameters $W / L=0.1, \rho_{\mathrm{e}} / \rho_{\mathrm{c}}=0.6, \rho_{\mathrm{p}} / \rho_{\mathrm{c}}=200$, $b / L=0.005$. The solid and dashed lines are symmetric and antisymmetric modes respectively.

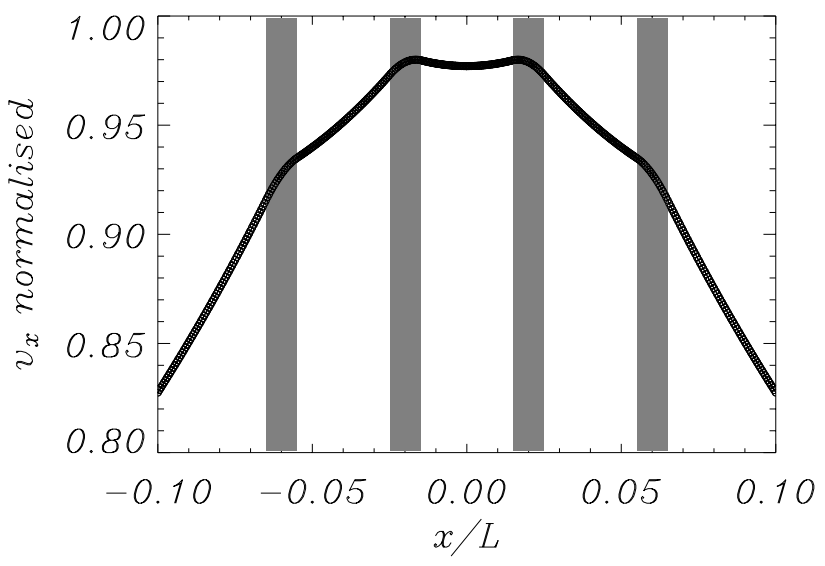

Fig. 10. Cut in the direction $z=0$ for the first symmetric mode for the parameters $W / L=0.1, \rho_{\mathrm{e}} / \rho_{\mathrm{c}}=0.6, \rho_{\mathrm{p}} / \rho_{\mathrm{c}}=200, b / L=0.005$ and $l / L=0.015=3 b / L$ for a system with four fibrils. A small $x$-range has been selected for a better rendition of the structure around the fibrils (shaded regions). 


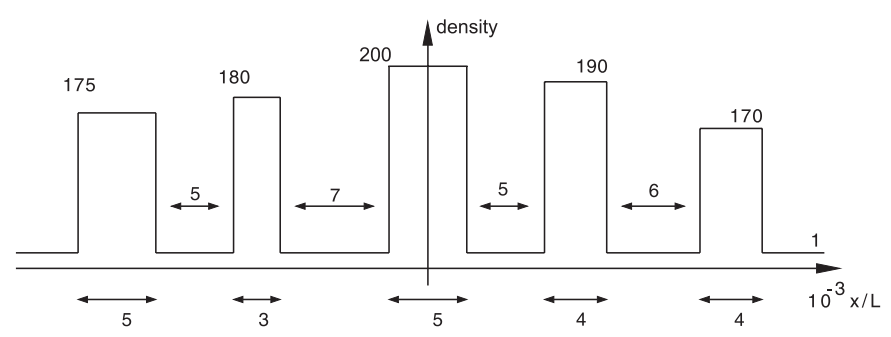

Fig. 11. Sketch of a cut in the direction $z=0$ of the normalised density profile $\left(\rho / \rho_{\mathrm{c}}\right)$ in the case of an non-homogeneous multifibril system. The normalised numerical values of the density are printed in each fibril, while the coronal medium has a value of 1 . The fibril width and the separation between them are also marked.

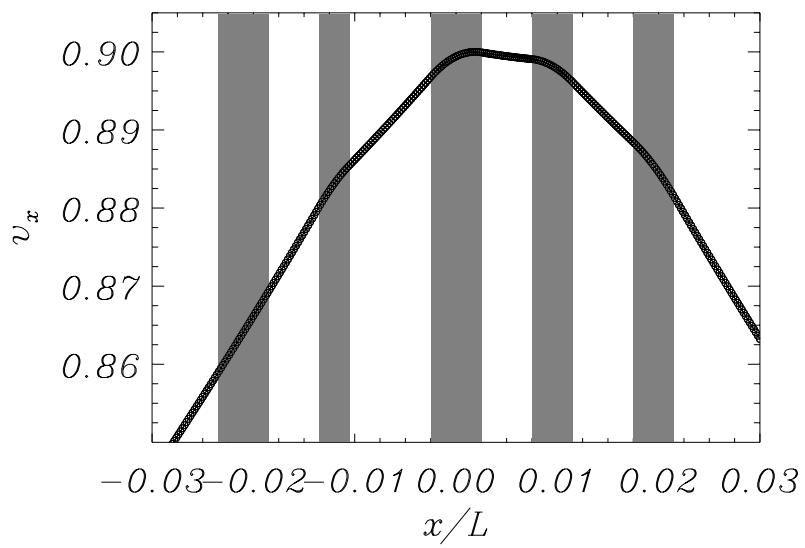

Fig. 12. Detail of the zone around the fibrils in a cut in the direction $z=0$ for the symmetric mode $\left(\omega L / c_{\mathrm{A}}=1.407\right)$ of the system of non-identical fibrils presented in Fig. 11. The fibril regions have been shadowed.

easily overcome their separation and, as a result, there is only one even non-leaky mode: the one with all the fibrils oscillating in phase. The overall structure is like the one in Fig. 8a, while the fine structure around the fibrils is that plotted in Fig. 12. The spatial profile of the velocity is similar to that of the densest fibril oscillating alone, with small contributions from the less dense fibrils.

Now we study the frequency dependence on the fibril separation. We have chosen to increase the fibril separation (named $l_{\text {ref }}$ ) keeping the widths of the fibrils and the ratios of separation between them as in Fig. 12. The results are plotted in Fig. 13 and compared with the single dominant fibril oscillating alone (dotted line). In the limit $l_{\text {ref }} \rightarrow \infty$, the structure and the frequencies of each fibril oscillating alone are recovered, while for small values of $l_{\text {ref }}$ all the non-phase oscillating modes are leaky, and only the mode described before remains, having a slightly smaller frequency than in the case of the single dominant fibril mode.

Also, looking at the spatial structure of the modes for separation $l_{\text {ref }} \rightarrow \infty$, we find that the spatial structure of each mode tends to be that of fibrils oscillating alone with their own frequency such as described in Paper I. For the first mode, all the fibrils oscillate in phase; for the second mode there are two groups of fibrils oscillating with opposition of phase between them; similarly, for the third mode there are three groups, for the fourth mode only two fibrils oscillate in phase (and the rest

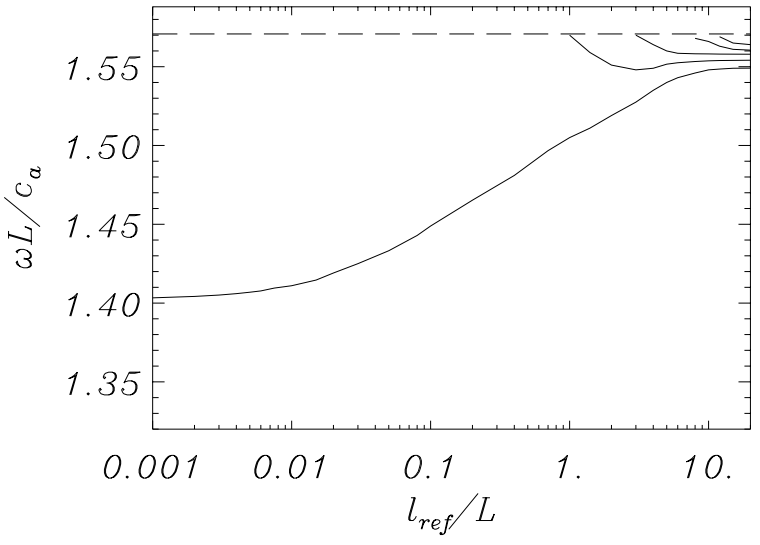

Fig. 13. Logarithmic plot of the dispersion relation for the multifibril system of Fig. 11. The range in the frequency axis has been selected smaller to better display the details. Notice that now we cannot identify modes in phase and in opposition of phase directly (except the fundamental one, which always corresponds to all the fibrils oscillating in phase, see main text).

in opposition of phase), and finally, for the fifth mode all the fibrils vibrate in opposition of phase with respect to their immediate neighbours. However, all these modes, except the fundamental one described in Fig. 12, are leaky in the realistic range $\left(l_{\text {ref }} \approx b\right)$.

Therefore, for realistic values of the separation between fibrils, the multifibril system oscillates in phase, with similar amplitudes and the same frequency (smaller than the eigenfrequency of the densest fibril), so if we looked at it with a broader scale, we would see something similar to Fig. 8a. Moreover, there would not be any trace of other fibril eigenmodes, at least in the stationary state.

\section{Conclusions}

Using the analytical and numerical procedure developed in Paper I, we have constructed a very simple model to study the interaction between fibrils when fast MHD are excited within them. The analytical results have been checked numerically in the simplest case, and both results are in a perfect agreement.

With the goal to mimic as accurately as possible real prominences, two different situations have been studied: homogeneous prominences, composed of fibrils with identical physical properties, and non-homogeneous prominences, in which the density of the considered fibrils is different, i.e. the Alfvén velocity is the parameter being modified.

When considering identical fibrils, the symmetric and antisymmetric modes of oscillation have been described, and the general result is that when two or more fibrils are considered, all the modes except the first symmetric one become leaky for realistic values of fibril separation. This fact has an important consequence: if a fibril is perturbed and starts to oscillate, only the stationary modes described in Paper I remain after a suitable time scale. However, in a system of fibrils, the symmetric and antisymmetric modes would be excited but, at the end, only the symmetric mode would remain, i.e. the fibrils would oscillate with the same frequency. Then, extrapolating these results 
to the whole prominence, after some time, the prominence fibrils would oscillate in spatial phase with the same frequency, so the prominence would seem to be an oscillating slab with its fine structure hardly discernible, but with the characteristic frequencies reflecting the fibril presence. Therefore, a group of these fibrils in a solar prominence would oscillate together with a frequency just slightly different form a slab without structure and width $n_{\mathrm{f}} 2 b$. If we continued this process for larger values of $n_{\mathrm{f}}$, we would tend to a periodic alternance of fibrils and coronal regions that could be studied with similar techniques to the ones used to describe a periodic array of slabs in Berton \& Heyvaerts (1987). This limit is left for further work.

In the case of non-identical fibrils, i.e. a non-homogeneous prominence, because of symmetry breaking there are no symmetric or antisymmetric solutions, and the most important conclusion is that when realistic separations among the fibrils of the system are considered, the fibrils oscillate in phase with slightly different amplitudes and with a frequency which is smaller than that of the densest fibril.

The effect of longitudinal propagation, similar to what was done for a single fibril in Díaz et al. (2003), remains to be studied. It is expected that the interaction becomes weaker, but for realistic values of the parameters there are only slight changes. If the longitudinal wavenumber $k_{y}$ is high enough, some antisymmetric modes could also be trapped.

On the other hand, there is the possibility to drain some energy from the excited fibril (apart from the leaky modes not studied here) and transfer it to its partners. The results presented here seem to suggest that after some time all the fibrils of a prominence region would be oscillating in phase with the same frequency. This could explain why groups of fibrils seem to oscillate together ( $\operatorname{Lin} 2004)$.

Finally, this study needs to be extended to more realistic geometries. It has been shown that the geometry has a very strong influence on the structure of the eigenmodes (Díaz et al. 2002), so one must be careful when applying these conclusions to a real solar prominence. In this regard, this work is only a first step for further models in this direction. A next step would to study the interaction of two identical cylindrical fibrils; for such a system of two fibrils, the modes of a single fibril would be shifted and a new array of modes would appear due to the lack of symmetry in the azimuthal direction. Then, the collective behaviour exhibited by packed cylindrical flux tubes could be considered and thus the results of the multifibril Cartesian systems studied in this paper could be extended.

Acknowledgements. A. J. Díaz, R. Oliver and J. L. Ballester acknowledge the financial support received from the Spanish MCyT under grant AYA2003-00123. A. J. Díaz also thanks the Spanish MCyT for grant BFM2000-1329.

\section{References}

Arregui, I., Oliver, R., \& Ballester, J. L. 2001, A\&A, 369, 1122 Arregui, I., Oliver, R., \& Ballester, J. L. 2003, A\&A, 402, 1129 Ballester, J. L., \& Priest, E. R. 1989, A\&A, 225, 213

Berton, R., \& Heyvaerts, J. 1987, Sol. Phys., 109, 201

Degenhardt, U., \& Deinzer, W. 1993, A\&A, 278, 288

Díaz, A. J., Oliver, R., Erdélyi, R., \& Ballester, J. L. 2001, A\&A, 379, 1083 (Paper I)

Díaz, A. J., Oliver, R., \& Ballester, J. L. 2002, ApJ, 580, 550

Díaz, A. J., Oliver, R., \& Ballester, J. L. 2003, A\&A, 402, 781

Edwin, P. M., \& Roberts, B. R. 1982, Sol. Phys., 76, 239

Engvold, O. 1976, Sol. Phys., 49, 283

Engvold, O. 2001, in INTAS Workshop on MHD Waves in Astrophysical Plasmas, ed. J. L. Ballester, \& B. Roberts, Universitat de les Illes Balears, Spain

Engvold, O., Kjeldseth-Moe, O., Bartoe, J. D. F., \& Brueckner, G. 1987, in Proc. 21st ESLAB Symp., ESA SP-275, 21

Joarder, P. S., Nakariakov, V., \& Roberts, B. 1997, Sol. Phys., 173, 81

Lin, Y. 2004, Ph.D. Thesis, Univ. of Oslo

Lin, Y., Engvold, O., van der Voort, L. R., Wiik, J. E., \& Berger, T. E. 2005, Sol. Phys., submitted

Menzel, D. H., \& Evans, J. W. 1953, Convegno Volta, 11, 119

Roberts, B. 1991, in Advances in Sol. System Magnetohydrodynamics, ed. E. R. Priest, \& A. W. Hood (Cambridge Press), 110

Schmieder, B., \& Mein, P. 1989, Hvar Obs. Bulletin 13, IAU Colloq., 119, 31

Schmieder, B., Raadu, M., \& Wiik, J. E. 1991, A\&A, 252, 353

Schmitt, D., \& Degenhardt, U. 1996, in Rev. Mod. Astron., ed. G. Klare (Springer-Verlag)

Yi, Z., \& Engvold, O. 1991, Sol. Phys., 134, 275

Yi, Z., Engvold, O., \& Keil, S. L. 1991, Sol. Phys., 132, 63 